\title{
Distinct Profile Differences in Subjective Cognitive Decline in the General Public Are Associated with Metacognition, Negative Affective Symptoms, Neuroticism, Stress, and Poor Quality of Life
}

\author{
Amy Jenkins $^{\mathrm{a}, *}$, Jeremy Tree ${ }^{\mathrm{b}}$ and Andrea Tales ${ }^{\mathrm{a}}$ \\ ${ }^{a}$ Centre for Innovative Ageing, Swansea University, Wales, UK \\ ${ }^{\mathrm{b}}$ Department of Psychology, Swansea University, Wales, UK
}

Accepted 22 January 2021

Pre-press 23 February 2021

\begin{abstract}
.
Background: Subjective cognitive decline (SCD) is increasingly recognized in both the clinical and research arenas as a risk factor for mild cognitive impairment (MCI) and dementia. Although SCD is etiologically heterogeneous and potentially treatable, in comparison to MCI and Alzheimer's disease, SCD remains poorly characterized with its clinical relevance often questioned.

Objective: This study's aim was to improve the characterization of SCD within the general public.

Methods: Individuals with SCD were compared to those without via a battery of measures.

Results: Both the SCD and the non-SCD group correlational analysis identified significant relationships between worse SCD, worse metacognitive dysfunction, negative affective symptoms, and greater levels of stress. The SCD group displayed additional correlational relationships between Cognitive Change Index (Self report) (CCI-S) scores, higher neuroticism scores, and poorer quality of life (QoL). Partial correlation analysis in the SCD group suggests CCI-S scores, anxiety, depression, and metacognition are intercorrelated. Ad hoc analyses using metacognition as the grouping variable found that those experiencing worse metacognitive dysfunction were significantly more likely to experience poorer SCD, psychological and social QoL, greater levels of anxiety, depression, stress, and neuroticism.

Conclusion: The emerging pattern from the analysis indicates that SCD appears associated with sub-clinical negative affective difficulties, metacognitive, and other psycho-social issues, and poorer QoL. Dysfunctional cognitive control at a meta-level may impact someone's ability to rationally identify cognitive changes, increase worry about cognitive changes, and allow such changes to impact their lives more than those with superior metacognitive control. Findings could impact SCD assessment, monitoring, early intervention, and ultimately reducing risk of further decline.
\end{abstract}

Keywords: Alzheimer's disease, anxiety, dementia, depression, metacognition, neuroticism, quality of life, stress, subjective cognitive decline

\footnotetext{
${ }^{*}$ Correspondence to: Dr. Amy Jenkins, College of Human and Health Sciences, Centre for Innovative Ageing, Haldane, Swansea University, Singleton Park, Swansea, SA2 8PP, UK. Tel.: +1 44 01792 205678; E-mail: A.Jenkins@swansea.ac.uk.
}

\section{INTRODUCTION}

Subjective cognitive decline (SCD) is evident prior to the onset of clinical impairment, is based on selfreporting, and could function as a specified group for trials on early intervention [1]. SCD is increasingly recognized in both the clinical and research arena as 
a risk factor for mild cognitive impairment (MCI) and dementia, particularly Alzheimer's disease (AD) [2]. However, compared to MCI and AD, SCD remains relatively poorly characterized and appears etiologically heterogeneous, with debate continuing regarding its clinical relevance and importance in relation to dementia.

Various terms have been introduced prior to SCD, namely subjective cognitive impairment; subjective memory complaints; subjective cognitive complaints; subjective memory impairment. They are often used interchangeably resulting in a lack of specificity, confusion, and heterogeneity [2]. For this research, the term SCD will be used for cognitively unimpaired subjects experiencing subjective cognitive changes relative to previous performance levels. Jessen et al. (2014) suggested this more appropriate term to reflect the neurodegenerative decline associated with $\mathrm{AD}$ [2]. Thus, they created a specific term and common concept to facilitate research, enable comparability, and synergy throughout the research literature. Due to subjective complaints being etiologically heterogeneous and unspecific, the Subjective Cognitive Decline Initiative (SCD-I) was created to help develop a common research framework for characterizing SCD, thus subjective complains due to pre-clinical AD [2]. SCD-I highlighted specific variables which should be considered SCD plus variables. Hence, variables that indicate an increased risk of developing $\mathrm{AD}$ such as a subjective decline in memory, rather than other domains of cognition, age at onset of $\mathrm{SCD} \geq 60$ years, onset of SCD within the last 5 years, confirmation of cognitive decline by an informant, and concerns/worries associated with SCD [2].

SCD may, for some individuals, represent individual differences of the normal aging process. What is in fact normal aging for a particular individual experiencing symptoms of SCD can often be considered as 'senior moments' [3], thus moments considered to be not of serious consequences and often regarded humorous. Past research has suggested that SCD can also be due to reversible causes such as anxiety and depression [4, 5], thus potentially modifiable risk factors [6]. Consequently, treating the potentially reversible causes of SCD could result in the removal or reduction in severity of SCD symptoms and improve healthy aging, and in some instances, reduce overall risk of developing dementia in the long term $[2,6,7]$. Therefore, the factors contributing to SCD may be more heterogeneous and multifaceted than previously and commonly envisaged, for instance, negative affective symptoms. Furthermore, irrespective of etiology, SCD can be detrimental to quality of life (QoL), self-perceived health $[8,9]$, and self-perceived workplace performance [7], and could contribute to restrictions in mobility and social activities [10,11].

Further research is required to identify SCD causality, characteristics, consequences, and possible treatments [10]. It is imperative to identify whether such factors could provide clarity on potentially reversible causes or neurodegenerative etiology.

\section{Subjective cognitive decline, negative affective symptoms, stress, neuroticism, metacognition, and poor quality of life}

Although debate continues with respect to cause and effect, increasing evidence is indicative of links between SCD and negative affective symptoms (anxiety and depression), lower self-efficacy, the reporting of health troubles, less perceived control of life's difficulties, and poorer QoL [8, 9, 12-19]. Less often investigated is the relationship between SCD, neuroticism, metacognition, and levels of stress. To address this lack of knowledge, these factors will be further investigated in this study. Furthermore, factors such as affective disturbances, chronic stress, sleep disturbances, could also be the cause, contributory factors or consequence of cognitive impairment; however, they could also lead, in turn, to further and even accelerated cognitive decline [18, 20]. It is imperative to identify if low mood/depression is associated with experiences of SCD [21]. Furthermore, Millan et al. (2012) found that QoL, work, and social functioning were severely compromised when cognitive deficits were poorly controlled in those who were experiencing depression at the time, and those who were in remission [22].

\section{Negative affective symptoms}

The most commonly identified concurrent conditions in older adults have been found to be depression and cognitive impairment [5]. Dementia risk increases when adverse factors such as depression, anxiety, and stress are present [23]. Donovan et al. (2014) identified that affective symptoms such as depression and anxiety are possible predictors of progression to further cognitive decline such as MCI [24]. Furthermore, approximately a third of worldwide dementia cases may be due to possible modifiable risk factors [6]. While the relationship between SCD with 
depression is well supported [25,26], the causality of depressive symptoms in SCD is difficult to determine [10].

Several studies have also proposed that SCD is associated with anxiety [13]. Luck et al. (2014) identified that SCD, specifically worrisome SCD, is predictive of neurodegenerative decline to dementia [4]. Jessen et al. (2014) also proposed that the relationship between SCD (defined by memory performance on delayed verbal recall) and AD risk was dependent on whether or not the individuals with SCD were reporting concern about it [27]. Thus, SCD was not predictive of AD in those individuals who were not concerned about it suggesting it corresponded more to age-associated decline rather than AD [27]. Therefore, those who were concerned were more likely to be experiencing memory impairment which may be different from normal aging. These findings support a previous study focused on memory which compared to individuals with SCD no concerns, those with SCD concerns were at double the risk of developing AD [28]. Evidently, there is a relationship between negative affective symptoms and SCD and therefore negative affective symptoms should not be ignored in the assessment and exploration of cognition. Thus, research studies should code for depression and anxiety but not include them in study exclusion criteria [2].

\section{Stress, neuroticism, and metacognition}

Elfgren and colleagues (2010) investigated the impact of psychosocial stress on participants with SCD (specifically memory decline), MCI and dementia [29]. They found that psychosocial stress (i.e., stress relating to psychological factors and surrounding social environment) was significantly more prevalent in the SCD group (71\% of cases) than the MCI group (18\%) and dementia group (0\%). They proposed that psychosocial stress interferes with the participants' evaluation of SCD and may in fact disturb the memory processes. However, at a three-year follow-up they found that in the SCD group only $14 \%$ reported the presence of psychosocial stress compared to $71 \%$ at baseline therefore highlighting the changeable nature of stress and the impact it can have.

Often cited in previous research is the relationship between objectively identifiable cognitive impairment (i.e., MCI and/or AD) and the personality trait neuroticism. Evidence suggests that it mediates the relationship between SCD (specifically memory decline) and psychological and physical health prob- lems [14]. Furthermore, Hill et al. (2019) identified that neuroticism was positively related to $S C D$, thus those with SCD were more likely to be neurotic compared to those without [30].

The self-perception of cognitive functioning, specifically SCD, could be explained by drawing on the idea of metacognition, thus individual's knowledge, understanding, and beliefs of their own cognitive functioning [31]. Shimamura (2000) postulates that metacognition is the control and evaluation of cognitive functioning [32]. It can be argued that SCD can be considered a form of metacognitive monitoring. Wells (2011) proposes that metacognition relates to beliefs about thinking, or having knowledge of cognitions, with metacognitive thinking likened to an orchestra. To produce an overture there must be a music score and a conductor. Metacognition is the score and the conductor behind the thinking [33]. Youn et al (2019) found that older adults with SCD, displayed improved cognition and identified brain region changes as a result of a Meta Memory Training program (MMT). The positive impact on the brain regions is believed to relate to metacognition, thus they suggest the MMT had an impact on improving cognitive control [34]. Note, the present study measures metacognitive beliefs, monitoring, and judgements, not how accurate the participants are at assessing their own cognitive ability.

\section{Study aims}

The aim of the current study was to further characterize SCD in the general public with respect to general objectively measured cognitive functioning, anxiety, depression, QoL, stress, metacognition, and neuroticism. We hypothesized that in comparison to those without SCD, those with SCD will display worse metacognitive dysfunction, worse negative affective symptoms, worse levels of stress, and worse QoL, and will be more neurotic.

\section{METHODS}

\section{Participants}

An opportunity sample methodological approach was used. Males $(\mathrm{N}=29)$ and females $(\mathrm{N}=90)$ aged 55-70 years were recruited from the general public living in South Wales, UK (Number of participants $[\mathrm{N}]=119$; mean $[\mathrm{M}]$ age $=62.46$ years, standard deviation $[\mathrm{SD}]=3.65)$. The inclusion criteria are, the general public, in good to excellent general medical 
and mental health from a self-reported perspective, thus a Likert scale response to simple questions. The exclusion criteria are, those with a clinical diagnosis of MCI or dementia (self-report medical diagnosis), self-report of decline in cognitive ability which can be explained by a psychiatric or neurological disease, previous head injury, medical disorder, medication (prescribed and non-prescribed) known to the participant to influence cognition, or substance use. Participants were recruited via advertisement on social media, group email (i.e., 50+ Network), posters etc. This study was approved by Swansea University ethics review boards and written informed consent was obtained from all participants.

\section{Battery of measures}

Mean education level, ethnicity and family history are presented in Table 1. The test battery comprised: Cognitive Change Index (Self report) (CCI-S) to measure SCD symptoms [35], higher score reflects worse SCD; The World Health Organization Quality of Life (WHOQOL-BREF; 26 items, the shortened version of the WHOQOL-100) [36], higher score reflects better QoL; Hospital Anxiety and Depression Scale (HADS; 14 items, 7 for anxiety and 7 for depression) [37], higher score reflects poorer anxiety and/or depression; The Big Five Inventory (BFI; 44 item scale) to measure neuroticism [38], higher score reflects an individual being more neurotic; Montreal Cognitive Assessment (MoCA; 30 items) [39], higher score reflects better cognitive functioning; The National Adult Reading Test (NART; 50 irregular words) to measure predicted IQ [40], higher score reflects higher predicted IQ; The MetaCognitions Questionnaire 30 (MCQ-30; 30 items) to measure metacognitive beliefs, monitoring tendencies, and judgement [41], higher score reflects greater metacognitive dysfunction; The Perceived Stress Scale (PSS; 10 items) [42], higher score reflects poorer perceived stress levels.

The sample was split based on their CCI-S test scores into the SCD group and the non-SCD group to determine any between-group differences or withingroup relationships. The CCI-S measure has one set of validated cut-off values to use as a method of grouping, which are the ones used within the wellestablished Alzheimer's Disease Neuroimaging Initiative (ADNI) study [43]. These cut-offs are based on the first 12 CCI-S items (out of 20) which relate to perceived memory concerns. Therefore, individuals are believed to be experiencing memory concerns if they score 20 or above on the first 12 items of the CCI-S. Figure 1A and 1B show the distribution of scores on the CCI-S measure across both the SCD and non-SCD group.

\section{Statistical analysis}

Overall, based on Kolmogorov-Smirnov analysis the data (i.e., on the CCI-S, MoCA, WHOQOLBREF, HADS, PSS, MCQ-30, and the BFI) were not normally distributed despite score transformations, therefore non-parametric analysis was applied (see Supplementary Table 1). To determine differences between the SCD and non-SCD group, the MannWhitney test was employed for continuous data and the Chi Square test was employed for categorical data. Furthermore, correlations, thus within-group relationships, between CCI-S scores with all test battery scores (and partial correlations) were analyzed using non-parametric Spearman's correlation coefficient. Hypothesized a priori predictions were made and therefore one-tailed analyses were run and reported below on variables previously investigated [12], and two-tailed analyses for those that have not.

\section{RESULTS}

As can be seen in Table 1, no significant participant characteristic group differences were identified in age, gender, years in full-time education, ethnicity, family history in cognitive impairment, general health, predicted IQ via NART scores, and general cognitive functioning via MoCA scores. Significant group differences were identified in participants CCI$\mathrm{S}$ scores, thus the scores used to divide the groups [31].

Subjective cognitive decline, general cognitive functioning, depression, anxiety, QoL, neuroticism, metacognition, and stress

\section{Between-group differences}

There were no significant group differences in performance on general cognitive functioning, physical health QoL, and social relationship QoL sub-scales (all $p$ values $>0.05$ ) between the SCD and non-SCD groups (Table 2). The $p$ value for physical health $\mathrm{QoL}$ was $p=0.052$ and therefore only slightly below the cut-off for significance. However, there were significant differences between these two groups regarding psychological and environmental QOL. There were also significant differences on anxiety, depression, 
Table 1

Participant characteristics in both the SCD and the non-SCD group

\begin{tabular}{|c|c|c|c|c|}
\hline & SCD & Non-SCD & $\begin{array}{l}\text { Pearson Chi Square Test: } \\
\text { corresponding categories in } \\
\text { the SCD \& non-SCD group }\end{array}$ & $\begin{array}{c}\text { Mann-Whitney Test: } \\
\text { corresponding categories } \\
\text { in the SCD \& non-SCD group }\end{array}$ \\
\hline Total N & 44 & 75 & - & - \\
\hline Age mean $(\mathrm{SD})[\mathrm{y}]$ & $62.16(3.68)$ & $62.64(3.65)$ & - & $\mathrm{NS} \mathrm{U}=1497.0(\mathrm{Z}=-0.846), p=0.398$ \\
\hline Gender N $(\%)$ & $\begin{array}{l}34 \text { (77.3) Female } \\
10 \text { (22.7) Male }\end{array}$ & $\begin{array}{l}56 \text { (74.7) Female } \\
19 \text { (25.3) Male }\end{array}$ & $\mathrm{NS} \chi^{2}(1, \mathrm{~N}=119)=0.102, p=0.749$ & - \\
\hline $\begin{array}{l}\text { Years in FT } \\
\text { Education mean (SD) }\end{array}$ & $15.16(2.84)$ & $15.03(2.62)$ & - & NS U $=1621.5(Z=-0.159), p=0.874$ \\
\hline Ethnicity group N (\%) & $\begin{array}{c}\text { Welsh - } 27(61.4) \\
\text { English - } 7(15.9) \\
\text { British/other - } 10(22.7)\end{array}$ & $\begin{array}{c}\text { Welsh - 48 (64) } \\
\text { English - } 12(16) \\
\text { British/other - 15 (20) }\end{array}$ & $\mathrm{NS} \chi^{2}(2, \mathrm{~N}=119)=0.129, p=0.938$ & - \\
\hline $\begin{array}{l}\text { Family history } \\
\text { of cognitive } \\
\text { impairment } \mathrm{N}(\%)\end{array}$ & $\begin{array}{c}\text { Yes (maternal) }-17(38.6) \\
\text { Yes (paternal) }-2(4.5) \\
\text { None }-25(56.8)\end{array}$ & $\begin{array}{c}\text { Yes (maternal) }-31(41.3) \\
\text { Yes (paternal) }-1(1.3) \\
\text { None }-43(57.3)\end{array}$ & $\mathrm{NS} \chi^{2}(2, \mathrm{~N}=119)=1.186, p=0.553$ & - \\
\hline $\begin{array}{l}\text { CCI-S full scale } \\
\text { mean }(\mathrm{SD})\end{array}$ & $36.23(7.39)$ & $23.84(3.19)$ & 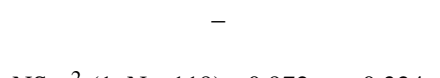 & $\begin{array}{c}\mathrm{U}=67.50(\mathrm{Z}=-8.739), p<0.001) \\
\text { Cohen's effect size: }-0.80 \text { Large }\end{array}$ \\
\hline General health (\%) & $\begin{array}{c}\text { Excellent }- \text { Good }=38(86.4) \\
\text { Fair }- \text { Poor }=6(13.6)\end{array}$ & $\begin{array}{l}\text { Excellent }- \text { Good }=69(92) \\
\quad \text { Fair }- \text { Poor }=6(8)\end{array}$ & $\mathrm{NS} \chi^{2}(1, \mathrm{~N}=119)=0.972, p=0.324$ & - \\
\hline $\begin{array}{l}\text { NART errors } \\
\text { mean (SD) }\end{array}$ & $8.11(5.63)$ & $7.85(5.40)$ & - & $\mathrm{NS} \mathrm{U}=1603.0(\mathrm{Z}=-0.260), p=0.795$ \\
\hline MoCA mean (SD) & $28.41(1.28)$ & $28.29(1.26)$ & & $\mathrm{NS} \mathrm{U}=1563.0(\mathrm{Z}=-0.493), p=0.622$ \\
\hline
\end{tabular}


A.

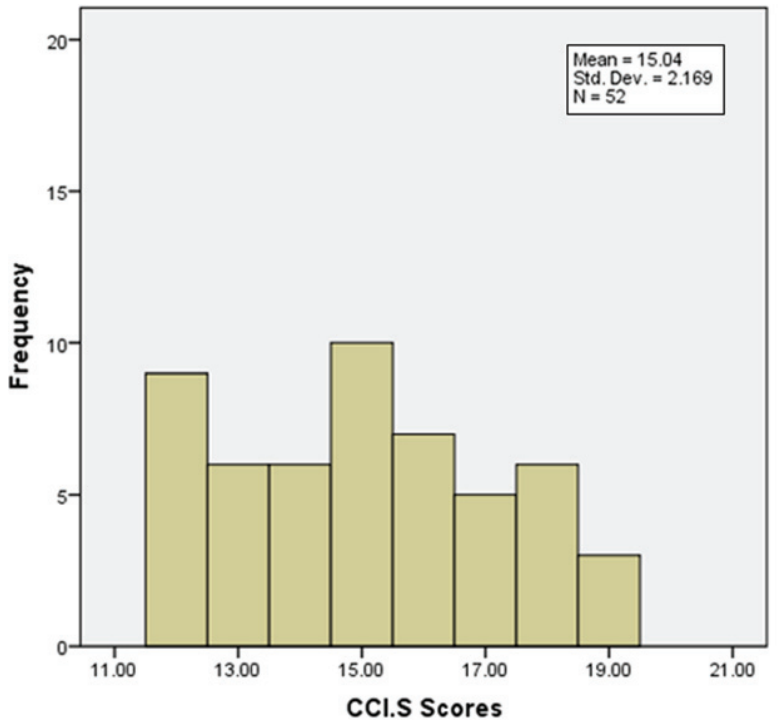

B.

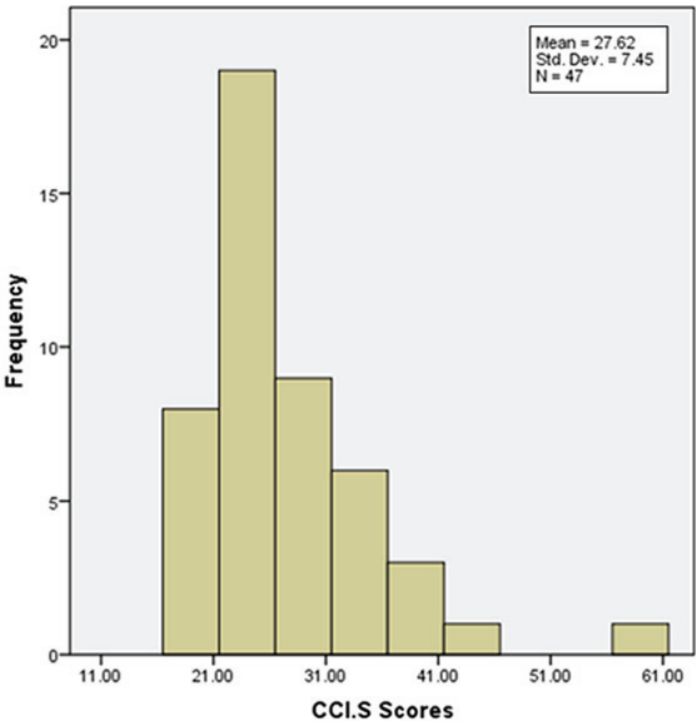

Fig. 1A. Distribution of CCI-S scores within the non-SCD group. B. Distribution of CCI-S scores within the SCD group.

Table 2

Between-group differences

\begin{tabular}{|c|c|c|c|}
\hline & $\begin{array}{c}\text { SCD } \\
\text { Mean (SD) }\end{array}$ & $\begin{array}{l}\text { Non-SCD } \\
\text { Mean (SD) }\end{array}$ & $\begin{array}{l}\text { Statistics: Mann- } \\
\text { Whitney U test }\end{array}$ \\
\hline $\mathrm{MoCA}$ & $28.41(1.28)$ & $28.29(1.26)$ & $\mathrm{U}=1563.0(\mathrm{Z}=-0.493), p=0.622$ \\
\hline $\begin{array}{l}\text { Physical Health QoL } \\
\text { (WHOQOL-BREF) }\end{array}$ & $15.93(2.95)$ & $16.76(2.75)$ & $\mathrm{U}=1302.5(\mathrm{Z}=-1.943), p=0.052$ \\
\hline $\begin{array}{l}\text { Social relationships QoL } \\
\text { (WHOQOL-BREF) }\end{array}$ & $15.05(3.21)$ & $15.59(3.28)$ & $\mathrm{U}=1453.5(\mathrm{Z}=-1.093), p=0.274$ \\
\hline $\begin{array}{l}\text { Psychological QoL } \\
\text { (WHOQOL-BREF) }\end{array}$ & $14.77(2.54)$ & $16.01(2.42)$ & $\begin{array}{c}\mathrm{U}=1119.5(\mathrm{Z}=-2.955), p=0.003 \\
\text { Cohen's effect size: }-0.27 \text { small }\end{array}$ \\
\hline $\begin{array}{l}\text { Environmental QoL } \\
\text { (WHOQOL-BREF) }\end{array}$ & $17.43(1.68)$ & $18.15(2.96)$ & $\begin{array}{c}\mathrm{U}=1226.5(\mathrm{Z}=-2.377), p=0.017 \\
\text { Cohen's effect size: }-0.22 \text { small }\end{array}$ \\
\hline Anxiety (HADS) & $5.86(3.00)$ & $3.80(2.71)$ & $\begin{array}{l}\mathrm{U}=956.0(\mathrm{Z}=-3.843), p<0.001 \\
\text { Cohen's effect size: }-0.35 \text { medium }\end{array}$ \\
\hline Depression (HADS) & $3.52(2.98)$ & $2.28(2.56)$ & $\begin{array}{c}\mathrm{U}=1185.0(\mathrm{Z}=-2.603), p=0.009 \\
\text { Cohen's effect size: }-0.24 \text { small }\end{array}$ \\
\hline Neuroticism (BFI) & $20.70(6.36)$ & $18.19(5.74)$ & $\begin{array}{c}\mathrm{U}=1264.5(\mathrm{Z}=-2.126), p=0.034 \\
\text { Cohen's effect size: }-0.19 \text { small }\end{array}$ \\
\hline Stress (PSS) & $15.11(7.99)$ & $8.96(5.44)$ & $\begin{array}{l}\mathrm{U}=850.50(\mathrm{Z}=-4.408), p<0.001 \text {; } \\
\text { Cohen's effect size: }-0.40 \text { medium }\end{array}$ \\
\hline Metacognition (MCQ-30) & $49.77(8.78)$ & $43.37(7.49)$ & $\begin{array}{l}\mathrm{U}=959.0(\mathrm{Z}=-3.807), p<0.001 \\
\text { Cohen's effect size: }-0.35 \text { medium }\end{array}$ \\
\hline
\end{tabular}

neuroticism, stress, and metacognition (Table 2). Overall, the SCD group demonstrated significantly poorer perceived psychological and environmental QoL, greater levels of anxiety and depression, neuroticism, stress, and poorer scores on metacognitive dysfunction compared to the non-SCD group.

\section{Within-group relationships}

In the non-SCD group, there were no significant within-group correlations between CCI-S scores and general cognitive functioning, neuroticism, and QoL. However, there were significant positive withingroup correlations between CCI-S scores and anxiety, depression, stress, and metacognition (Table 3). In the SCD group, there were no significant withingroup correlations between CCI-S scores and general cognitive functioning, and environmental QoL. However, there were significant positive within-group correlations between CCI-S scores and neuroticism, anxiety, depression, stress, and metacognition, and significant negative within-group correlations with 
Table 3

Within-group correlations between CCI-S sores and all variables: Spearman's correlation coefficient

\begin{tabular}{lcc}
\hline & \multicolumn{1}{c}{ Non-SCD } & SCD \\
\hline MoCA & $r_{\mathrm{s}}=-0.44, p(1$-tailed $)=0.355$ & $r_{\mathrm{s}}=-0.19, p(1$-tailed $)=0.114$ \\
Physical Health QoL (WHOQOL-BREF) & $r_{\mathrm{s}}=-0.17, p(1$-tailed $)=0.15$ & $r_{\mathrm{s}}=-0.40, p(1$-tailed $)=0.004$ \\
Social Relationships QoL (WHOQOL-BREF) & $r_{\mathrm{s}}=-0.00, p(1$-tailed $)=0.98$ & $r_{\mathrm{s}}=-0.26, p(1$-tailed $)=0.046$ \\
Psychological QoL (WHOQOL-BREF) & $r_{\mathrm{s}}=-0.09, p(1$-tailed $)=0.43$ & $r_{\mathrm{s}}=-0.48, p(1$-tailed $)<0.001$ \\
Environmental QoL (WHOQOL-BREF) & $r_{\mathrm{s}}=-0.13, p(1$-tailed $)=0.27$ & $r_{\mathrm{s}}=-0.16, p(1$-tailed $)=0.149$ \\
Anxiety (HADS) & $r_{\mathrm{s}}=0.28, p(1$-tailed $)=0.007$ & $r_{\mathrm{s}}=0.27, p(1$-tailed $)=0.037$ \\
Depression (HADS) & $r_{\mathrm{s}}=0.34, p(1$-tailed $)=0.002$ & $r_{\mathrm{s}}=0.39, p(1$-tailed $)=0.005$ \\
Neuroticism (BFI) & $r_{\mathrm{s}}=0.11, p(1$-tailed $)=0.173$ & $r_{\mathrm{s}}=0.38, p(1$-tailed $)=0.006$ \\
Stress (PSS) & $r_{\mathrm{s}}=0.24, p(2$-tailed $)=0.039$ & $r_{\mathrm{s}}=0.30, p(2$-tailed $)=0.051$ \\
Metacognition (MCQ-30) & $r_{\mathrm{s}}=0.27, p(2$-tailed $)=0.021$ & $r_{\mathrm{s}}=0.30, p(2$-tailed $)=0.045$ \\
\hline
\end{tabular}

physical QoL, psychological QoL, and social QoL, (Table 3).

Due to the multiple comparisons being investigated in some of the correlational analyses, the Bonferroni correction method was used to control for the possibility of type one errors. After Bonferroni correction on the QoL sub-scales ( $\alpha$ level $0.05 / 4=0.012$ ), the only significant within-group correlations to remain were in relation to psychological $(p<0.001)$ and physical $(p=0.004)$ QoL, not Social QoL $(p=$ 0.046).

To identify possible unique variance between two of the variables in the SCD group, a third variable of interest would need to be controlled for by conducting a partial correlation. As a result of past research indicating a clear link between anxiety, depression, and metacognition (e.g., [31]), the relationship between these variables and SCD were investigated. In the SCD group, there was no association between subjects CCI-S scores and metacognition while controlling for anxiety $(\mathrm{r}(41)=0.259, p=0.093)$, depression $(\mathrm{r}(41)=0.252, p=0.103)$, or neuroticism ( $\mathrm{r}$ $(41)=0.224, p=0.149)$. However, when controlling for metacognition, there was an association between subjects CCI-S scores and depression $(\mathrm{r}(41)=0.352$, $p=0.021)$, and between CCI-S scores and neuroticism ( $\mathrm{r}(41)=0.317, p=0.038)$, but no association between CCI-S scores and anxiety $(\mathrm{r}(41)=0.220$, $p=0.156$ ). The partial correlation analyses were rerun to control for age, years in education, and gender in addition to anxiety, depression, and neuroticism. No association was still found between subjects CCI$\mathrm{S}$ scores and metacognition while controlling for anxiety $(\mathrm{r}(38)=0.185, p=0.254)$, depression $(\mathrm{r}(38)=$ $0.242, p=0.132$ ), and neuroticism $(\mathrm{r}(38)=0.206$, $p=0.202)$. When controlling for metacognition, age, years in education, and gender, there was still an association between CCI-S scores and depression

Table 4

Metacognition as a grouping variable, between-group differences

\begin{tabular}{|c|c|c|c|}
\hline & $\begin{array}{c}\text { Metacognition } \\
\text { high }(\mathrm{N}=20) \\
(\text { Mean and SD) }\end{array}$ & $\begin{array}{c}\text { Metacognition } \\
\text { low }(\mathrm{N}=20) \\
\text { (Mean and } \mathrm{SD})\end{array}$ & $\begin{array}{l}\text { Mann-Whitney: } \\
\text { between-group } \\
\text { differences }\end{array}$ \\
\hline$\overline{\mathrm{MoCA}}$ & $27.90(1.25)$ & $28.70(1.13)$ & $\mathrm{NS}(\mathrm{U}=131.50(\mathrm{Z}=-1.913), p=0.056)$ \\
\hline $\begin{array}{l}\text { Physical Health QoL } \\
\text { (WHOQOL-BREF) }\end{array}$ & $16.50(2.59)$ & $17.75(1.59)$ & $\mathrm{NS}(\mathrm{U}=146.50(\mathrm{Z}=-1.481), p=0.139)$ \\
\hline $\begin{array}{l}\text { Social Relationships QoL } \\
\text { (WHOQOL-BREF) }\end{array}$ & $14.25(3.61)$ & $16.85(2.51)$ & $\begin{array}{c}\mathrm{U}=117.50(\mathrm{Z}=-2.258), p=0.024 \\
(\text { Cohen's effect size: }-0.36 \text { medium })\end{array}$ \\
\hline $\begin{array}{l}\text { Psychological QoL } \\
\text { (WHOQOL-BREF) }\end{array}$ & $14.20(2.73)$ & $16.95(1.76)$ & $\begin{array}{l}\mathrm{U}=76.50(\mathrm{Z}=-3.384), p<0.001 \\
(\text { Cohen's effect size: }-0.54 \text { large })\end{array}$ \\
\hline $\begin{array}{l}\text { Environmental QoL } \\
\text { (WHOQOL-BREF) }\end{array}$ & $18.20(4.38)$ & $18.40(1.82)$ & $\mathrm{NS}(\mathrm{U}=150.50(\mathrm{Z}=-1.372), p=0.170)$ \\
\hline Anxiety (HADS) & $6.50(3.65)$ & $2.55(1.64)$ & $\begin{array}{l}\mathrm{U}=65.50(\mathrm{Z}=-3.665), p<0.001 \\
\text { (Cohen's effect size: }-0.58 \text { large) }\end{array}$ \\
\hline Depression (HADS) & $3.75(3.43)$ & $1.30(1.42)$ & $\begin{array}{l}\mathrm{U}=106.50(\mathrm{Z}=-2.582), p=0.010 \\
(\text { Cohen's effect size: }-0.41 \text { medium })\end{array}$ \\
\hline Neuroticism (BFI) & $22.95(6.60)$ & $15.50(4.84)$ & $\begin{array}{l}\mathrm{U}=74.00(\mathrm{Z}=-3.415), p<0.001 \\
(\text { Cohen's effect size: }-0.54 \text { large })\end{array}$ \\
\hline Stress (PSS) & $14.00(7.62)$ & $6.95(4.32)$ & $\begin{array}{c}\mathrm{U}=90.00(\mathrm{Z}=-2.982), p=0.003 \\
(\text { Cohen's effect size: }-0.47 \text { medium })\end{array}$ \\
\hline Metacognition (MCQ-30) & $59.30(5.90)$ & $34.20(2.53)$ & $\begin{array}{l}\mathrm{U}=0.00(\mathrm{Z}=-5.421), p<0.001 \\
(\text { Cohen's effect size: }-0.86 \text { large })\end{array}$ \\
\hline
\end{tabular}

NS, not significant. 
( $\mathrm{r}(38)=0.417, p=0.007)$, however the association between CCI-S scores and neuroticism was no longer significant when age, years in education, and gender were also controlled for $(\mathrm{r}(38)=0.291, p=0.069)$. The association between CCI-S scores and anxiety remained non-significant when controlling for age, gender, and years in education, as well as metacognition $(\mathrm{r}(38)=0.218, p=0.176)$.

\section{Ad-hoc analysis}

Ad-hoc analyses were run to build on the initial main aims of this research and gain further insight into the initial research findings and potential profiles of SCD. Thus, due to past research regarding the association between metacognition, anxiety and depression (e.g., [31]), and the findings from the present study's initial analysis, it was decided that additional group-difference analyses would be run on all variables employing metacognition as the grouping variable. Participants with the highest 20 scores and the lowest 20 scores on the MCQ-30 were grouped and analyzed across all study variables. The rounded threshold of 20 participants was selected so that the overall size of the two groups was of similar size to the study's SCD group $(\mathrm{N}=44)$ while ensuring the MCQ-30 scores were distinct. Table 4 shows the means and standard deviations for each variable and whether significant group differences were identified. Those with higher scores of metacognition, thus metacognitive dysfunction, were significantly more likely to experience poor scores on SCD, anxiety, depression, stress, neuroticism, psychological and social QoL.

\section{DISCUSSION}

The aim of the current study was to further characterize SCD (in the general public) with respect to general objectively measured cognitive functioning, anxiety, depression, QoL, stress, metacognition, and neuroticism. The results of the analysis indicate that there are significant differences between those older adults who are experiencing SCD compared to those who are not. Specifically, individuals with SCD experienced higher levels of anxiety, depression, and stress; displayed poorer scores on psychological and environmental QoL; poorer levels of metacognitive dysfunction; and were more likely to be neurotic. In the SCD group, all these variables were also found to be significantly correlated with CCI-S scores therefore indicating a possible relationship with the participants subjective changes in cognition. Interestingly, irrespective of the significant betweengroup differences, stress, metacognition, anxiety, and depression were also significantly correlated with CCI-S scores in the non-SCD group. This result could be explained by the fact that not all those experiencing difficulties with negative affective symptoms, stress, and metacognitive dysfunction, are at a greater risk of experience SCD. The results of the partial correlation analysis suggest that anxiety, depression, and metacognition are inter-correlated, thus they seem to all be related to one another. Intercorrelation is assumed due to both the pattern of statistical findings (i.e., the impact on scores when controlling for each variable) and past research [30]. Such a presumption provides strength to the theory that particular profiles of individuals are more at risk of experiencing SCD than others. It must be noted, however, that the average scores for anxiety and depression were all within the 'normal' range, therefore the negative affective symptoms being experienced are sub-clinical. Therefore, it is proposed that their negative affective symptoms do not have to be identifiable at a clinical level to potentially have an impact on one's health, wellbeing, and cognitive functioning.

There has been much debate over the years regarding whether negative affective symptoms for example should be identified as a cause or consequence of cognitive changes, however, causality is very difficult to determine. SCD is etiologically heterogeneous and found to be related to for instance normal aging [2], depression [25, 26], anxiety [13], chronic fatigue [44], schizophrenia [45], and bipolar disorder [46].

Irrespective of directional causation, changes to cognitive functioning, whether that be subjectively or objectively recognized, can still be both a personal concern as well as a public health concern [47], and can detrimentally influence QoL and self-perceived health $[8,9]$, cause emotional distress and poor wellbeing [9], and fear of significant underlying brain pathology and impending dementia [7,2]. To identify cause and effect was not possible in this study, and therefore we are unable to propose that for instance SCD is caused by anxiety, depression, stress, neuroticism, and metacognitive dysfunction.

The consistent strength and pattern of statistical findings in the core analysis of this study, particularly the significant group-differences, suggest that there are distinct profiles of individuals experiencing SCD in comparison to those who are not. Such profiles could be explained by the impact of metacognitive dysfunction thus, poor cognitive control at a 
meta-level is likely to have an impact on for instance negative affective symptoms. Therefore, ad hoc analyses were run using metacognition as the grouping variable. The metacognitive based group differences were distinct and indicated certain profiles of individuals. Thus, the group of individuals with higher scores of metacognition, indicating metacognitive dysfunction, were significantly more anxious, depressed, stressed, neurotic, experiencing poor psychological and social QoL. Importantly, they were also experiencing significantly poorer scores on SCD. Consequently, this suggests that those experiencing metacognitive dysfunction may not only be more likely to identify SCD, but also more likely to worry about it and allow it to impact on their lives to a greater extent than those with superior metacognitive control. As previously noted, the relationship between metacognition, anxiety, and depression are likely inter-correlated and therefore all impact on one another. Also, it must be noted that the present study measures metacognitive beliefs, monitoring, and judgements, not how accurate the participants are at assessing their own cognitive ability.

Past research suggests that an individual's knowledge, understanding, and beliefs of their own memory and cognitive functioning could impact on SCD [31]. Shimamura (2000) postulates that metacognition is the control and evaluation of cognitive functioning. It can be argued that the self-evaluation of SCD can be considered a form of metacognitive monitoring [32]. For example, an individual may evaluate their memory by making a judgement regarding something they have learned. Shimamura (2000) proposed that such monitoring is likely to therefore influence metacognitive control. For instance, in the above judgement on learning, the individual may evaluate that they did not learn something very well and therefore would allocate more time to learning it again.

Zanardo et al. (2006) put forward the idea that metacognition is multidimensional, thus based on memory monitoring; memory attributional style; factual knowledge of how the memory functions and knowledge of memory strategies; memory related self-efficacy beliefs; and memory related influences such as the impact of depression and anxiety [31]. These dimensions provide a possible explanation for the initial and ad hoc findings within this study. For instance, in relation to the dimension of memory related self-efficacy beliefs, thus memory ability, selfassessment, and feelings towards changes to memory. The evaluation of one's memory and self-efficacy beliefs are believed to be related to age; hence older adults were perceived to be more pessimistic about their memory ability [48]. Zanardo et al. (2006) suggested that a self-fulfilling prophecy is the reason why some older adults have a negative perception and poorer level of self-efficacy of their memory ability. Thus, they propose some older adults perceive their memory ability based on what other people think of them [31].

Arguably, such a metacognitive theoretical perspective should be considered in the translation from research into clinical practice. Such a perspective encapsulates the findings from both the initial and ad hoc analyses in the present study. For instance, poor perception of cognitive ability seems to be the result of a more global metacognitive process involving the influence of many variables such as mood, anxiety, QoL, self-efficacy, neuroticism, stress, and metacognitive monitoring. The complex interplay of multiple variables such as these will likely impact on general health. For example, higher levels of selfefficacy are related to more engagement in behaviors that are regarded as healthy (i.e., keeping physically and mentally fit) [49]. Poor health-related behavior is related to low mood, higher levels of anxiety, and cognitive decline [50]. From a clinical and intervention perspective, Zanardo et al. (2006) proposed that improving older adults' self-efficacy and attributional style of their cognitive functioning is possible by encouraging them to have more confidence in their cognitive capabilities and their ability to accurately judge their cognition [31]. Furthermore, such improvements could encourage individuals to engage more in health-related behaviors, and ultimately improve SCD, metacognitive functioning, mood, anxiety, stress, etc.

Such theoretical and research evidence provides a possible explanation for the initial and ad hoc results found in this study. Thus, those in the current study with SCD also had higher levels of anxiety, depression, stress, neuroticism, and poor physical and psychological QoL. Consequently, we hypothesize that symptoms of SCD may be contributed to by a combination of excessive rumination, worry, and negative bias towards threats (i.e., cognitive decline). The results from the ad hoc analyses also suggest profile differences possibly characterized by levels of metacognition.

\section{Study limitations and future research}

A potential limitation of this study is its crosssectional design. In relation to all variables measured, 
this study was the first testing phase in a planned longitudinal study. Most of the sample agreed to be contacted again in the future to be re-tested in order to identify any possible changes over time. Importantly, past research has identified that psychosocial stress for instance was significantly more prevalent in the SCD group compared to those with MCI or dementia [29]. Participants were tested again after three-years and they found that in the SCD group, only $14 \%$ reported the presence of psychosocial stress compared to $71 \%$ at baseline therefore highlighting the changeable nature of stress and the impact it can have. Findings such as this not only support the need for a longitudinal study to re-test the participants, but also the need for variables such as metacognition to be measured at baseline and follow-up in clinical and research settings, and that cognition should not be tested and appraised in isolation. Consequently, this could give rise to future research into causality, thus intervention-based studies on metacognitive therapy and SCD. An additional study limitation is the lack of established causal links between the variables. Further research would need to be carried out to identify such links.

To conclude, in response to the research, theoretical associations, and effective intervention evidence, it is plausible to suggest that metacognition and negative affective symptoms play an important role in the characterization of SCD. Furthermore, having a better understanding of SCD characteristics could inform clinical interventions to improve symptoms of $\mathrm{SCD}$. This study is a valuable contribution to research regarding the characterization of SCD profiles in the general population. The sample reported poorer scores in relation to metacognitive dysfunction, negative affective symptoms, stress, psychological and environmental QoL, but not social and physical QoL. Therefore, regardless of whether the SCD is the cause or consequence of such distress, their cognitive changes and concerns should not be dismissed as benign. Suitable SCD monitoring could enable potentially reversible indications to be treated (e.g., metacognitive dysfunction), thus not only with the aim to alleviate symptoms of SCD but also to reduce further risk of cognitive decline [21].

\section{DISCLOSURE STATEMENT}

Authors' disclosures available online (https:// www.j-alz.com/manuscript-disclosures/20-0882r3).

\section{SUPPLEMENTARY MATERIAL}

The supplementary material is available in the electronic version of this article: https://dx.doi.org/ 10.3233/JAD-200882.

\section{REFERENCES}

[1] Smart CM, Segalowitz SJ, Mulligan BP, Koudys J, Gawryluk JR (2016) Mindfulness training for older adults with subjective cognitive decline: Results from a pilot randomized controlled trial. J Alzheimers Dis 52, 757-774.

[2] Jessen F, Amariglio RE, Van Boxtel M, Breteler M, Ceccaldi M, Chételat G, Duboisk, Carole Dufouill, Kathryn A. Ellis, van der Flier WM, Glodzik L, van Harten AC, de Leon MJ, McHugh P, Mielke MM, Molinuevo JL, Mosconi L, Osorio RS, Perrotin A, Petersen RC, Rabin RA, Rami L, Reisberg B, Rentz DM, Sachdev PS, de la Sayette V, Saykin AJ, Scheltens P, Shulman MB, Slavin MJ, Sperling RA, Stewart R, Uspenskaya O, Vellas B, Visser PJ, Wagner M (2014) A conceptual framework for research on subjective cognitive decline in preclinical Alzheimer's disease. Alzheimers Dement 10, 844-852.

[3] Desai AK, Schwarz L (2011) Subjective cognitive impairment: When to be concerned about 'senior moments'. Curr Psychiatry 10, 31-44.

[4] Luck T, Riedel-Heller SG, Luppa M, Wiese B, Bachmann C, Jessen F, Bickel H, Weyerer S, Pentzek M, König HH, Prokein J, Eisele M, Wagner M, Mösch E, Werle J, Fuchs A, Brettschneider C, Scherer M, Breitner JC, Maier W (2014) A hierarchy of predictors for dementia-free survival in oldage: Results of the AgeCoDe study. Acta Psychiatr Scand 129, 63-72.

[5] Diniz BS, Butters MA, Albert SM, Dew MA, Reynolds CF (2013) Late-life depression and risk of vascular dementia and Alzheimer's disease: Systematic review and metaanalysis of community-based cohort studies. Br J P sychiatry 202, 329-335.

[6] Norton S, Matthews FE, Barnes DE, Yaffe K, Brayne C (2014) Potential for primary prevention of Alzheimer's disease: An analysis of population-based data [published correction appears in Lancet Neurol. 2014 Nov;13(11):1070]. Lancet Neurol 13, 788-794.

[7] McIntyre RS, Soczynska JZ, Woldeyohannes HO, Alsuwaidan, MT, Cha DS, Carvalho AF, Jerrell JM, Dale RM, Gallaugher LA, Muzina DJ, Kennedy SH (2015) The impact of cognitive impairment on perceived workforce performance: Results from the International Mood Disorders Collaborative Project. Compr Psychiatry 56, 279-282.

[8] Mol M, Carpay M, Ramakers I, Rozendaal N, Verhey F, Jolles J (2007) The effect of perceived forgetfulness on quality of life in older adults; a qualitative review. Int J Geriatr Psychiatry 22, 393-400.

[9] Montejo P, Montenegro, M, Fernandez MA, Maestu F (2011) Subjective memory complaints in the elderly: Prevalence and influence of temporal orientation, depression and quality of life in a population-based study in the city of Madrid. Aging Ment Health 15, 85-97.

[10] Hill NL, Mogle JM, Munoz E, Wion R, Colancecco EM (2015) Assessment of subjective cognitive impairment among older adults. J Gerontol Nurs 41, 28-35. 
[11] Amariglio RE, Townsend MK, Grodstein F, Sperling RA, Rentz DM (2011) Specific subjective memory complaints in older persons may indicate poor cognitive function. $\mathrm{J} \mathrm{Am}$ Geriatr Soc 59, 1612-1617.

[12] Jenkins A, Tree J, Thornton IM, Tales A (2019) subjective cognitive impairment in 55-65-year-old adults is associated with negative affective symptoms, neuroticism, and poor quality of life. J Alzheimers Dis 67, 1367-1378.

[13] Clarnette RM, Almeida OP, Forstl H, Paton A, Martins RN (2001) Clinical characteristics of individuals with subjective memory loss in Western Australia: Results from a crosssectional survey. Int J Geriatr Psychiatry 16, 168-174.

[14] Comijs HC, Deeg DJ, Dik MG, Twisk JW, Jonker C (2002) Memory complaints; the association with psycho- affective and health problems and the role of personality characteristics. A 6-year follow-up study. J Affect Disord 72, 157-167.

[15] Yochim BP, Mueller AE, Segal DL (2013) Late life anxiety is associated with decreased memory and executive functioning in community dwelling older adults. $J$ Anxiety Disord 27, 567-575.

[16] Beaudreau SA, Hara R (2008) Late-life anxiety and cognitive impairment: A review. Am J Geriatr Psychiatry 16, 790-803.

[17] Gallacher J, Bayer A, Fish M, Pickering J, Pedro S, Dunstan F, Ebrahim S, Ben-Shlomo Y (2009) Does anxiety affect risk of dementia? Findings from the Caerphilly Prospective Study. Psychosom Med 71, 659-666.

[18] Innes KE, Selfe TK, Khalsa DS, Kandati S (2016) Effects of meditation versus music listening on perceived stress, mood, sleep, and quality of life in adults with early memory loss: A pilot randomized controlled trial. J Alzheimers Dis 52, 1277-1298.

[19] Lee H, Park S, Lim K, Park Y, Jang J (2016) Association between lifestyle and cognitive impairment among women aged 65 years and over in the Republic of Korea. Educ Gerontol 42, 198-208.

[20] Panza F, Introno A, Colacicco AM, Capurso C, Del Parigi A, Caselli RJ... Solfrizzi V (2009) Temporal relationship between depressive symptoms and cognitive impairment: The Italian Longitudinal Study on Aging. J Alzheimers Dis 17, 899.

[21] Yoo I, Woo JM, Lee SH, Fava M, Mischoulon D, Papakostas GI, Kim EJ, Chung S, Ha JH, Jeon HJ (2015) Influence of anxiety symptoms on improvement of neurocognitive functions in patients with major depressive disorder: A 12-week, multicenter, randomized trial of tianeptine versus escitalopram, the CAMPION study. J Affect Disord 185, 24-30.

[22] Millan MJ, Agid Y, Brüne M, Bullmore ET, Carter CS, Clayton NS, Connor R, Davis S, Deakin B, DeRubeis RJ, Dubois B, Geyer MA, Goodwin GM, Gorwood P, Jay TM, Joëls M, Mansuy IM, Meyer-Lindenberg A, Murphy D, Rolls E, Saletu B, Spedding M, Sweeney J, Whittington M, Young LJ (2012) Cognitive dysfunction in psychiatric disorders: Characteristics, causes and the quest for improved therapy. Nat Rev Drug Discov 11, 141-168.

[23] Ju YE, McLeland JS, Toedebusch CD, Xiong C, Fagan AM, Duntley SP, Morris JC, Holtzman DM (2013) Sleep quality and preclinical Alzheimer disease. JAMA Neurol 70, 587593.

[24] Donovan NJ, Amariglio RE, Zoller AS, Rudel RK, GomezIsla T, Blacker D, Hyman BT, Locascio JJ, Johnson KA, Sperling RA, Marshall GA (2014) Subjective cognitive concerns and neuropsychiatric predictors of progression to the early clinical stages of Alzheimer disease. Am J Geriatr Psychiatry 22, 1642-51.
[25] Grambaite R, Hessen E, Auning E, Aarsland D, Selnes P, Fladby T (2013) Correlates of subjective and mild cognitive impairment: Depressive symptoms and CSF biomarkers. Dement Geriatr Cogn Dis Extra 3, 291.

[26] Chen ST, Siddarth P, Ercoli LM, Merrill DA, Torres-Gil F, Small GW (2014) Modifiable risk factors for Alzheimer disease and subjective memory impairment across age groups. PLoS One 9, e98630.

[27] Jessen F, Wolfsgruber S, Wiese B, Bickel H, Mösch E, Kaduszkiewicz H, Pentzek M, Riedel-Heller SG, Luck T, Fuchs A, Weyerer S, Werle J, van den Bussche H, Scherer M, Maier W, Wagner M; German Study on Aging, Cognition and Dementia in Primary Care Patients (2014) AD dementia risk in late MCI, in early MCI, and in subjective memory impairment. Alzheimers Dement 10, 76-83.

[28] Jessen F, Wiese B, Bachmann C, Eifflaender-Gorfer S, Haller F, Kölsch H, Luck T, Mösch E, van den Bussche H, Wagner M, Wollny A, Zimmermann T, Pentzek M, RiedelHeller SG, Romberg HP, Weyerer S, Kaduszkiewicz H, Maier W, Bickel H; German Study on Aging, Cognition and Dementia in Primary Care Patients Study Group (2010) Prediction of dementia by subjective memory impairment: Effects of severity and temporal association with cognitive impairment. Arch Gen Psychiatry 67, 414-422.

[29] Elfgren C, Gustafson L, Vestberg S, Passant U (2010) Subjective memory complaints, neuropsychological performance and psychiatric variables in memory clinic attendees: A 3-year follow-up study. Arch Gerontol Geriatr 51, e110e114.

[30] Hill NL, Mogle J, Bhargava S, Bell TR, Wion RK (2019) The influence of personality on memory self-report among black and white older adults. PLoS One 14, e0219712

[31] Zanardo F, De Beni R, Moè A (2006) Influence of otherbeliefs on self-beliefs and on everyday memory self-report in the elderly. Aging Clin Exp Res 18, 425-432.

[32] Shimamura AP (2000) Toward a cognitive neuroscience of metacognition. Conscious Cogn 9(2 Pt 1), 313-323; discussion 324-326.

[33] Wells A (2011) Metacognitive therapy for anxiety and depression. Guilford Press.

[34] Youn JH, Ryu SH, Lee JY, Park S, Cho SJ, Kwon H, Yang JJ, Lee JM, Lee J, Kim S, Livingston G (2019) Brain structural changes after multi-strategic metamemory training in older adults with subjective memory complaints: A randomized controlled trial. Brain Behav 9, e01278.

[35] Saykin AJ, Wishart HA, Rabin LA, Santulli RB, Flashman LA, West JD, McHugh TL, Mamourian AC (2006) Older adults with cognitive complaints show brain atrophy similar to that of amnestic MCI. Neurology 67, 834-842.

[36] (1998) Development of the World Health Organization WHOQOL-BREF quality of life assessment. The WHOQOL Group. Psychol Med 28, 551-558.

[37] Zigmond AS, Snaith RP (1983) The hospital anxiety and depression scale. Acta Psychiatr Scand 67, 361-370.

[38] John OP, Donahue EM, Kentle RL (1991) The big five inventory-versions $4 a$ and 54. Institute of Personality and Social Research, University of California, Berkeley, Berkeley, CA.

[39] Nasreddine ZS, Phillips NA, Bédirian, V, Charbonneau S, Whitehead V, Collin, I, Cummings JL, Chertkow H (2005) The Montreal Cognitive Assessment, MoCA: A brief screening tool for mild cognitive impairment. J Am Geriatr Soc 53, 695-699.

[40] Nelson HE, Willison J (1991) National Adult Reading Test (NART). Nfer-Nelson Windsor. 
[41] Wells A, Cartwright-Hatton S (2004) A short from of the metacognitions questionnaire: Properties of the MCQ-30. Behav Res Ther 42, 385-396.

[42] Cohen S, Kamarck T, Mermelstein R (1983) A global measure of perceived stress. J Health Soc Behav 24, 386-396.

[43] Risacher SL, Kim S, Nho K, Foroud T, Shen L, Petersen RC, Jack CR Jr, Beckett LA, Aisen PS, Koeppe RA, Jagust WJ, Shaw LM, Trojanowski JQ, Weiner MW, Saykin AJ (2015) APOE effect on Alzheimer's disease biomarkers in older adults with significant memory concern. Alzheimers Dement 11, 1417-1429.

[44] Jason LA, Torres-Harding SR, Jurgens A, Helgerson J (2004) Comparing the Fukuda et al. criteria and the Canadian case definition for chronic fatigue syndrome. J Chronic Fatigue Syndr 12, 37-52.

[45] Homayoun S, Nadeau-Marcotte F, Luck D, Stip E (2011) Subjective and objective cognitive dysfunction in schizophrenia - is there a link? Front Psychol 2, 148.
[46] Jensen JH, Støttrup MM, Nayberg E, Knorr U, Ullum H, Purdon SE, Kessing LV, Miskowiak KW (2015) Optimising screening for cognitive dysfunction in bipolar disorder: Validation and evaluation of objective and subjective tools. J Affect Disord 187, 10-19.

[47] Reisberg B, Shulman MB, Torossian C, Leng L, Zhu W (2010) Outcome over seven years of healthy adults with and without subjective cognitive impairment. Alzheimers Dement 6, 11-24.

[48] Hess TM (1990) Aging and cognition: Knowledge organization and utilization (Vol. 71). Elsevier.

[49] Conner M, Norman P (2005) Predicting health behaviour. McGraw-Hill Education, UK.

[50] Elwood PC, Pickering J, Bayer A, Gallacher JEJ (2002) Vascular disease and cognitive function in older men in the Caerphilly cohort. Age Ageing 31, 43-48. 